\title{
Complex Interaction of Dendritic Connectivity and Hierarchical Patch Size on Biodiversity in River-Like Landscapes
}

\author{
Francesco Carrara, ${ }^{1, \star}$ Andrea Rinaldo, ${ }^{1,2}$ Andrea Giometto, ${ }^{1,3}$ and Florian Altermatt ${ }^{3}$ \\ 1. Laboratory of Ecohydrology, École Polytechnique Fédérale Lausanne (EPFL), 1015 Lausanne, Switzerland; 2. Dipartimento di \\ Ingegneria Civile, Edile e Ambientale (ICEA), Università di Padova, 35131 Padova, Italy; 3. Department of Aquatic Ecology, Swiss \\ Federal Institute of Aquatic Science and Technology (Eawag), 8600 Duebendorf, Switzerland
}

Submitted January 22, 2013; Accepted July 18, 2013; Electronically published November 27, 2013

Online enhancement: appendixes. Dryad data:http://dx.doi.org/10.5061/dryad.15np2.

\begin{abstract}
Habitat fragmentation and land use changes are causing major biodiversity losses. Connectivity of the landscape or environmental conditions alone can shape biodiversity patterns. In nature, however, local habitat characteristics are often intrinsically linked to a specific connectivity. Such a link is evident in riverine ecosystems, where hierarchical dendritic structures command related scaling on habitat capacity. We experimentally disentangled the effect of local habitat capacity (i.e., the patch size) and dendritic connectivity on biodiversity in aquatic microcosm metacommunities by suitably arranging patch sizes within river-like networks. Overall, more connected communities that occupy a central position in the network exhibited higher species richness, irrespective of patch size arrangement. High regional evenness in community composition was found only in landscapes preserving geomorphological scaling properties of patch sizes. In these landscapes, some of the rarer species sustained regionally more abundant populations better tracking their own niche requirements compared to landscapes with homogeneous patch size or landscapes with spatially uncorrelated patch size. Our analysis suggests that altering the natural link between dendritic connectivity and patch size strongly affects community composition and population persistence at multiple scales. The experimental results are demonstrating a principle that can be tested in theoretical metacommunity models and eventually be projected to real riverine ecosystems.
\end{abstract}

Keywords: dendritic ecological networks, riverine ecosystems, community assembly, directional dispersal, experimental microcosms, spatial heterogeneity.

\section{Introduction}

Biodiversity has been strongly affected by humans over the last decades. In many systems, diversity has been declining at all levels, with major consequences on ecosystem

* Corresponding author; e-mail: francesco.carrara@epfl.ch.

Am. Nat. 2014. Vol. 183, pp. 13-25. (C) 2013 by The University of Chicago. 0003-0147/2014/18301-54418\$15.00. All rights reserved.

DOI: $10.1086 / 674009$ functioning and services (Vörösmarty et al. 2010). Anthropogenic alterations of the natural environment, such as land use changes and habitat fragmentation, directly threaten species (Fahrig 2003; Poff et al. 2007; Franzen and Nilsson 2010; Gonzalez et al. 2011; Perkin and Gido 2012). One of the main challenges in community ecology and conservation biology is the understanding of the interactions between local and regional factors controlling population demography and community composition (Sheldon 1968; Chesson 2000; Hubbell 2001; Holyoak et al. 2005) and to eventually predict community dynamics (Fagan 2002; Economo and Keitt 2008).

Recent theoretical, experimental, and comparative work suggests that dispersal constrained by specific habitat structures is a major determinant of the observed diversity patterns at both species and genetic level (Fagan 2002; Muneepeerakul et al. 2008; Clarke et al. 2008; Morrissey and de Kerckhove 2009; Brown and Swan 2010; Finn et al. 2011; Carrara et al. 2012). Up to now, however, two major aspects have been neglected by most theoretical and experimental studies. First, landscape connectivity was generally considered independent of local environmental factors, such as habitat quality, patch size, environmental disturbances, and intra- and interspecific competition. While there are indeed cases for which this simplification is appropriate, such as forests (Hubbell 2001), island archipelagos (MacArthur and Wilson 1963), or natural ponds (Altermatt et al. 2008; De Bie et al. 2012), it does not represent many natural landscapes, such as fluvial and mountainous ecosystems, where local properties of the habitat and connectivity are intrinsically linked (Sheldon 1968; Benda et al. 2004; Lowe et al. 2006). Second, past studies generally adopted constant dispersal rates, symmetric kernels, and simplified landscape attributes (Warren 1996; Gonzalez et al. 1998; Mouquet and Loreau 2003; Matthiessen and Hillebrand 2006; Altermatt et al. 2011b; 
Chisholm et al. 2011). Traditionally, many studies in stream ecology, influenced by the river continuum concept (Vannote et al. 1980) have considered linear conceptual models to analyze drainage basins. Such simplified linear environmental matrices, however, may not completely capture biodiversity patterns within dendritic ecosystems (Fagan 2002; Grant et al. 2007; Brown and Swan 2010; Finn et al. 2011; Carrara et al. 2012).

In riverine ecosystems, landscape-forming discharges are related to total contributing drainage area, a by-product of spatial aggregation, depth, and width of the active river cross section (Leopold et al. 1964; Rodriguez-Iturbe and Rinaldo 1997; Benda et al. 2004). The river network not only provides suitable ecological corridors for individuals to disperse (Fagan 2002; Rodriguez-Iturbe et al. 2009) but often dictates the availability of microhabitats that species may eventually exploit (Cardinale 2011). Habitat capacity (i.e., river width/depth, reflecting patch size in rivers) scales with contributing area, dispersal is often biased downstream, and the distribution and intensity of disturbances are intrinsically linked with the position along the network through abrupt changes at confluences (Benda et al. 2004). Consequently, spatial correlations emerge between local properties and regional network descriptors in dendritic environments, where the hierarchical spatial organization of environmental heterogeneity is a fundamental driver of local species richness and community composition (Fernandes et al. 2004; Muneepeerakul et al. 2007). Riverine ecosystems, which are among the most threatened ecosystems on Earth (Vörösmarty et al. 2010), are thus a prominent natural system in which disentangling the effect of local environmental conditions and connectivity of the landscape on diversity is needed (Lowe et al. 2006; Lake et al. 2007; Gonzalez et al. 2011; Grant et al. 2012). For example, habitat capacity and interannual streamflow variability are changed in rivers undergoing hydropower development or cross-basin connections (Poff et al. 2007; Grant et al. 2012; Ziv et al. 2012). Furthermore, land-use and agricultural practices in many countries are affecting riparian zones, foreseing buffer zones on a fixed distance from the river bank only, irrespective of the spatial position within the river network (Gassner 2006). Theoretical, empirical, and comparative studies have suggested that the degradation of riparian vegetation structure and alteration of connectivity between the patches in the habitat mosaic may significantly reduce stream diversity at different trophic levels (Urban et al. 2006; Vörösmarty et al. 2010; Grant et al. 2012; Perkin and Gido 2012; Ziv et al. 2012). However, most studies on ongoing habitat change and legal regulations regarding riverine landscapes are not considering the intrinsic link of habitat capacity and network position (Lake et al. 2007).

Here, we experimentally singled out the interaction of dendritic connectivity and local habitat capacity (by modulating patch size) on the diversity of microorganisms in dendritic metacommunities, which were mimicking network structure and patch connectivity of natural river networks. Specifically, we singled out the individual influence of connectivity and habitat capacity on microbial diversity by using three different configurations of patch sizes (Riverine, Random, and Homogeneous), connected following a river network geometry (fig. 1; "Material and Methods"). In Riverine landscapes, local habitat capacity correlates with position along the network and distance to the outlet (fig. A1, table A1; figs. A1-A5 and tables A1-A5 available online). Larger downstream communities receive more immigrants from upstream communities, eventually having a combined positive effect on biodiversity. In the Homogeneous and Random landscapes, motivated by ongoing riverine habitat modifications, local habitat capacity (i.e., the patch size) does not preserve the geomorphological scaling observed in natural river systems (Leopold et al. 1964; Rodriguez-Iturbe and Rinaldo 1997). By measuring species' persistence and species' density we followed diversity patterns in terms of $\alpha$-, $\beta$-, and $\gamma$-diversity (local species richness, among-community dissimilarity, and regional species richness) and community evenness in the above landscape configurations. Aquatic microcosms as employed here and in several other studies (e.g., Fukami and Morin 2003; Cadotte et al. 2006; Haddad et al. 2008; Carrara et al. 2012; Giometto et al. 2013) offer a useful bridge between theoretical models and comparative field studies, to test for general macroecological principles (Holyoak and Lawler 2005; Livingston et al. 2012). Findings from such laboratory experiments, even if not directly comparable to natural systems, may cast light on important underlying mechanisms that steer metacommunity dynamics in river systems.

\section{Material and Methods \\ The Riverine Landscapes}

Each metacommunity (MC) consisted of 36 local communities grown in culture well-plates connected by dispersal. Communities, composed of nine protist and one rotifer species (see "Aquatic Communities"), were connected according to five different river network geometries (fig. 1). We used dendritic network landscapes derived from five different space-filling optimal channel networks (OCNs; Rinaldo et al. 1992; Rodriguez-Iturbe et al. 1992) known to reproduce the scaling properties observed in real river systems (Rinaldo et al. 2006). An appropriate coarsegraining procedure was enforced to reduce a complex construct to an equivalent $6 \times 6$-patch network, preserving the characteristics of the original three-dimensional basin 
A 'Riverine' landscapes

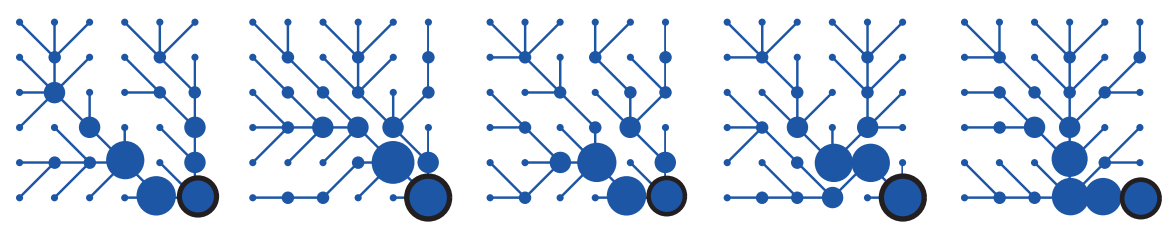

B 'Random' landscapes
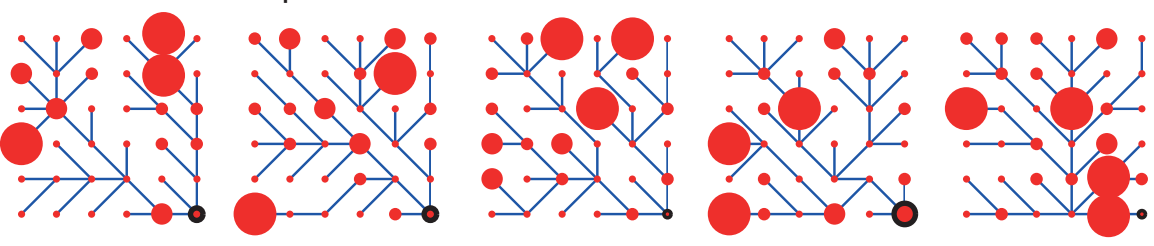

C 'Homogeneous' landscapes
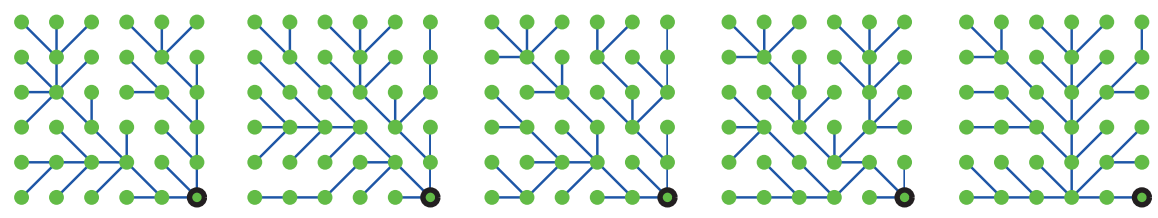

Figure 1: Spatial configuration of dendritic networks and corresponding patch sizes in the microcosm experiment. $A$, Riverine landscapes (blue) preserved the observed scaling properties of real river basins; $B$, Random landscapes (red) had the exact values of volumes as in the Riverine landscapes, randomly distributed across the networks; $C$, in Homogeneous landscapes (green) the total volume of the whole metacommunity was equally distributed to each 36 local communities. Patch size (size of the circle) is scaled to the actual medium volume. We had five unique river-like (dendritic) networks (columns: dispersal to neighboring communities followed the respective network structure, with a downstream bias in directionality toward the "outlet" community (black circled dot).

(for details, see app. A; apps. A-C available online). To have independent replication on the level of the networks, we have specifically chosen five different realization of river network configurations.

We relate the habitat capacity of an MC to physical properties that affect persistence of species (Reche et al. 2005). The largest organism to be sustained by an ecosystem is known to depend on habitat size (see, e.g., Banavar et al. 2007), termed "habitat capacity." Therefore, habitat capacity conceptualizes our ability to rank different landscapes in terms of their capacity to support viable populations. We implemented three different treatments of patch size configurations in each of the five OCN landscapes (fig. 1): (i) a "Riverine" landscape, in which the volume $v_{i}$ (i.e., the patch size) of the local community (LC) $i$ preserves the scaling law observed in real river systems: $v_{i} \propto A_{i}^{1 / 2}$. Area $A_{i}$ is the drainage area of the LC $i$, defined as the sum over all the volumes $v_{j}$ draining in that particular point; (ii) a "Random" landscape, in which the exact values of patch volumes $v_{i}$ as in the Riverine landscape were randomly distributed across the network; and (iii) a "Homogeneous" landscape where the total volume of the whole MC was equal to the other two treatments but each LC had a constant average value $\bar{v}_{\text {hom }}=$
$3.6 \mathrm{~mL}$ (Riverine $=$ Riv, Random $=$ Ran, Homogeneous $=$ Hom). We binned values of $v_{i}$, based on their original drainage area, in four size categories $(2,3.5,6$, and 12 $\mathrm{mL}$ ). In order to test species coexistence in isolation, we had 72 communities of "isolation" treatment, in which the patch sizes were equal to the first two replicates of the Riv configuration of the main experiment but without dispersal. Environmental conditions, dictated in the well plates by the ratio of surface area to volume, are changing between communities with different patch size, favoring different sets of species at a time (app. B, fig. B1; figs. B1, B2 available online).

\section{Aquatic Communities}

We kept LCs in multiwell culture plates containing a variable medium volume $(2-12 \mathrm{~mL})$, in a climatized room at $21^{\circ} \mathrm{C}$ under constant fluorescent light. Protozoan Pellets (Carolina Biological Supply) and soil provided nutrients for bacteria (Bacillus cereus, Bacillus subtilis, and Serratia marcescens), which are consumed by protists. Each LC within an MC initially contained nine protist species (Blepharisma sp., Chilomonas sp., Colpidium sp., Euglena gracilis, Euplotes aediculatus, Paramecium aurelia, Para- 
mecium bursaria, Spirostomum sp., and Tetrahymena sp.) and one rotifer species (Cephalodella sp., in the following included when speaking about protist; see app. B for species details). On day 0 , we added $K_{s} / 10$ individuals of each species, except for E. gracilis ( $K_{\mathrm{Eug}} / 100$ individuals), which naturally occurs at higher densities. Species' specific carrying capacities $K_{\mathrm{s}}$ and intrinsic growth rates $r_{\mathrm{s}}$ were measured in pure cultures (Altermatt et al. 2011a; Carrara et al. 2012).

\section{The Dispersal Events}

After the onset of the experiment, a dispersal event occurred every 3 days, in total eight times. Each time, half of the individuals of each community emigrated. Emigration happened by transfer of the individuals in the medium in well-mixed conditions and was thus density independent. The dispersers were manually transferred from every single LC to the nearest neighbors along the network, with absorbing boundary conditions. Emigration and subsequent immigration happened simultaneously across the landscape in well-mixed conditions. This specific type of density-independent (diffusive) dispersal, chosen to avoid long-tailed dispersal events, imposes equal per capita dispersal rates for all species. By applying eight one-step dispersal events, individuals from any population may disperse at most the average maximum size of our networks. In the dispersal procedure, we imposed a bias in directionality; that is, the probability for an individual to be dispersed into a downstream direction was double that of dispersing upstream. We introduced into the system a dispersal-induced mortality by discarding half of the emigrating individuals (equal to $25 \%$ of LC). We enforced the landscapes to maintain the initial spatial volume configuration by adding fresh medium to LC with a negative balance after the dispersal or by disregarding immigrating individuals in exceedance (see app. A for a detailed description of the method). Parallel to the experiment, we developed a suite of metacommunity models, including a purely diffusive model, maintaining the dispersal characteristics of the experiment and thus complementing and extending our experimental findings (app. A).

\section{Biodiversity Patterns}

On day 27, 3 days after the last dispersal-disturbance treatment, we measured species richness and species abundance (on a logarithmic scale) for each LC. This time interval corresponds to $\approx 10-100$ generations, depending on the species' intrinsic growth rate, and the dynamics occurs over ecologically significant timescales (Carrara et al. 2012). We screened the entire culture plate under a stereo microscope (Olympus SZX16) to avoid false absences of the rarer species. We then estimated species density following a standard procedure (Haddad et al. 2008; Altermatt et al. 2011b) by direct microscope observation of 0.5 $\mathrm{mL}$ of medium. We obtained the number of species present in the MC ( $\gamma$-diversity) and in every single LC ( $\alpha$-diversity) and the spatial distribution of $\alpha$-diversity. For $\beta$ diversity, we considered Jaccard's dissimilarity index, $\beta_{\mathrm{F}}$, based on species presence-absence, and an index based on species abundances, $\beta_{\mathrm{A}}$. Index $\beta_{\mathrm{J}}$ is defined as $1-$ $S_{i j} /\left(S_{i}+S_{j}-S_{i j}\right)$, where $S_{i j}$ is the number of species present in both local communities $i$ and $j$, whereas $S_{i}$ is the total number of species in LC $i$. Index $\beta_{\mathrm{A}}$ is defined as $\left(\sum_{k=1}^{S} w_{k}\left|x_{i k}^{\prime}-x_{j k}^{\prime}\right|\right) /\left(\sum_{k=1}^{S} w_{k}\right)-\beta_{\mathrm{J}}$, where $x_{j k}$ is the abundance of species $k$ in the LC $j, x_{j k}^{\prime}=\log x_{j k}+1$, whenever $x_{j k} \neq 0, w_{k}$ is a weight to take into account joint absences, and $S=10$ is the species pool. Index $\beta_{\mathrm{J}}$ only looks at compositional differences in species identities, whereas $\beta_{\mathrm{A}}$ studies the compositional differences in abundances, and is not bounded between 0 and 1 . Their sum gives the modified Gower's index, $\beta_{\mathrm{MG}}$ (Anderson et al. 2006). Thereby we could discriminate the effects of species richness on $\beta$-diversity. We measured local community evenness (LC evenness), described by $E_{i}=$ $1-2 / \pi \cdot \arctan \left[\sum_{s=1}^{S}\left(\log x_{i s}-\sum_{t=1}^{S} \log x_{i t} / S\right)^{2} / S\right]$. This defines an index bounded between 0 and 1, independent of species richness (Smith and Wilson 1996). Regional evenness was calculated from species' populations in the whole network.

\section{Statistical Analysis}

We used degree of connectivity $d$ and patch size $v$ as local network descriptors. Term $d_{i}$ is defined as the number of connected neighboring nodes to the LC $i$, whereas $v_{i}$ represent its habitat capacity. We used distance to the outlet $o$, drainage area $A$, and the ecological diameter $l$ as regional network descriptors. Term $o_{i}$ is calculated as the shortest path connecting $i$ to the outlet community. The specific spatial arrangement of patch sizes upstream of LC $i$ defines $A_{i}$. The average distance of $i$ from all other communities in the river network defines $l_{i}=\left\langle d_{i j}\right\rangle_{j}$, where $d_{i j}$ represents the shortest topological distance between $i$ and $j$. Thus, $l_{i}$ is the inverse of the closeness centrality of community $i$ (Newman 2010) and in riverine landscapes defines network positioning (fig. Al; table Al).

We performed an ANCOVA with configuration treatments as categorical fixed effects, OCNs as random effect, and the above-described network descriptors (continuous variables) as fixed effects. Normality and constancy of variance in $\alpha$-diversity and LC evenness over covariates were verified with Bartlett's test. A parallel analysis performed with a generalized linear mixed model (GLMM) framework was conducted, giving qualitatively consistent results. 
However, as model assumptions on error structure were better fulfilled by the ANCOVA than by a GLMM with Poisson error structure, we restricted ourselves to the former. Models were hierarchically simplified in a stepwise algorithm, starting with the full model and removing nonsignificant terms, with highest-level interactions first. We analyzed the overall effect of the configuration treatment on $\alpha$-diversity and LC evenness. We then used individual ANCOVAs within each landscape configuration to disentangle the effect of individual network properties on biodiversity indicators for each landscape configuration separately. We performed Kolmogorov-Smirnov tests (K$\mathrm{S}$ tests) on the cumulative density functions of $\alpha$ - and $\beta$ diversity and LC evenness of the three landscapes configurations. Mantel tests were used to study how $\beta$-diversity was influenced by topological distance, differences in environmental conditions and differences in network position in individual landscapes (Anderson et al. 2011). Differences in environmental conditions and network positioning were captured by respectively taking, for two focal communities $i$ and $j$, the absolute differences in patch sizes $\left|v_{i}-v_{j}\right|$ and ecological diameters $\left|l_{i}-l_{j}\right|$. The notation $\langle\cdot\rangle$ in the main text means a spatial average over the nodes, while ${ }^{-}$represents an average over the five landscape replicates.

\section{Results}

The spatial distribution of hierarchical patch size significantly affected community composition in dendritic environments (for all full ANCOVA results, see tables A2A5; data are deposited in the Dryad Digital Repository: http://dx.doi.org/10.5061/dryad.15np2; Carrara et al. 2013). The $\alpha$-diversity and LC evenness were significantly different across the three landscape configurations, Riv, Ran, and Hom (ANCOVA $\alpha$-diversity: $F_{2,4}=7.99, P=$ .0006, fig. $2 A$; ANCOVA LC evenness: $F_{2,4}=4.65, P=$ .01 , fig. $2 B$ ). Regionally, at the landscape scale, $\gamma$-diversity and mean $\alpha$-diversity did not vary significantly across the three different landscape configurations $\left(\bar{\gamma}_{\text {Riv }}=5.8\right.$, $\bar{\gamma}_{\text {Ran }}=6.2, \quad \bar{\gamma}_{\text {Hom }}=6, \quad\langle\bar{\alpha}\rangle_{\text {Riv }}=4.72, \quad\langle\bar{\alpha}\rangle_{\text {Ran }}=4.63$, $\langle\bar{\alpha}\rangle_{\text {Hom }}=4.83$; $P$ values of all pairwise K-S tests on different configuration treatments were $P>.1$ ). The LC evenness was higher for the configuration treatments in which spatial heterogeneity was introduced by varying patch sizes, that is, Riv (K-S test, $P<10^{-5}, K=0.33$ ) and Ran landscapes (K-S test, $P<10^{-5}, K=0.26$ ) compared to Hom landscapes (inset, fig. 2B). No significant difference was detected between Riv and Ran landscapes (K-S test, $P=.45, K=0.09$ ). The $\beta$-diversity (modified Gower's index $\beta_{\mathrm{MG}}$ ) was higher in the two heterogeneous landscape configurations (Riv, Ran) compared to the Hom landscapes (K-S tests, both $P<10^{-5}$; fig. $2 C$ ). The Ran land-
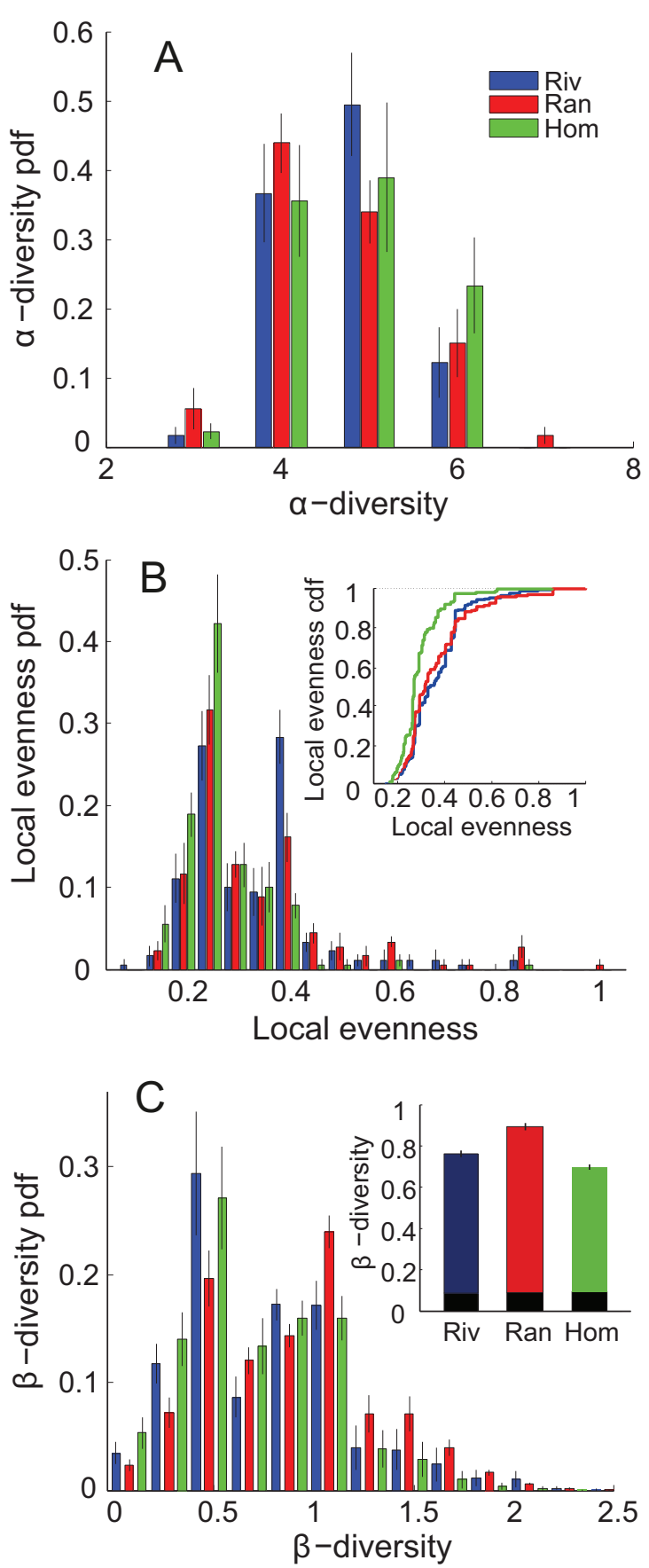

Figure 2: Probability density function (pdf) for $\alpha$-diversity (A), local evenness $(B)$, and $\beta$-diversity in Riverine (Riv; blue), Random (Ran; red), and Homogeneous (Hom; green) configurations over the five replicated dendritic landscapes. The insets give the cumulative density function of local evenness $(B)$ and the mean \pm SEM of $\beta$-diversity $(C)$, decomposed in an abundance-based index $\left(\beta_{\mathrm{A}}\right.$, upper coloredcoded part), and presence-absence-based index $\left(\beta_{\mathrm{J}}\right.$, black, bottom part). 
scapes showed the highest values of $\beta_{\mathrm{MG}}$ (K-S test, $P<$ $10^{-5}, K=0.16$ ), but considering only $\beta_{\mathrm{J}}$, no significant differences between the three configurations were found (inset, fig. 2C).

In the isolation treatment, species richness increased with increasing patch size. Larger protist species (especially Paramecium aurelia but also Paramecium bursaria) preferentially occupied patches with larger sizes (figs. B1, B2). The $\alpha$-diversity was significantly affected by the degree of connectivity $\left(F_{1,4}=77.5, P<10^{-5}\right.$; fig. $\left.3 A\right)$ and by patch size $\left(F_{1,4}=15.8, P=.001\right.$; fig. $\left.3 B\right)$. A significant interaction was observed between connectivity and patch size on $\alpha$-diversity $\left(F_{1,4}=12.05, P=.0006\right)$, whereas distance to the outlet, ecological diameter, and drainage area had no overall significant effect on $\alpha$-diversity. LC evenness was significantly affected by drainage area only $\left(F_{1,4}=\right.$ 11.49, $P=.005$; fig. $3 I$ ).

\section{$\alpha$-Diversity Patterns}

Because of the significant interaction between configuration treatment (i.e., Riv, Ran, and Hom) and individual network descriptors (tables A2, A3), three separate ANCOVA analyses were needed to address within-landscape relationships (one for each landscape configuration; table A4). In the following, we compare the three analyses (Riv, Ran, and Hom) separately for each network descriptor. The $\alpha$-diversity significantly increased with increasing degree of connectivity $\left(P_{\text {Riv }}=.002, P_{\text {Ran }}<10^{-5}, P_{\text {Hom }}=\right.$ $.005)$, although with different slopes $\left(\bar{m}_{\text {Riv }}=0.26\right.$; $\bar{m}_{\text {Ran }}=0.28 ; \bar{m}_{\text {Hom }}=0.40$; fig. $3 A$ ). Patch size significantly determined $\alpha$-diversity in Ran landscapes $\left(P_{\text {Ran }}=\right.$ .0002 ; fig. $3 B$ ), where it also interacted with connectivity $\left(P_{\text {Ran }}=.016\right)$. Distance to the outlet significantly affected $\alpha$-diversity in Riv and Hom landscapes $\left(P_{\text {Riv }}=.041\right.$; $\left.P_{\text {Hom }}=.021\right)$, where $\alpha$-diversity decreased with increasing distance to the outlet $\left(\bar{m}_{\mathrm{Riv}}=-0.22, \bar{m}_{\mathrm{Hom}}=-0.18\right.$; fig. $3 C)$. No dependence of $\alpha$-diversity on distance to the outlet was observed in the Ran landscapes $\left(\bar{m}_{\text {Ran }}=-0.022\right.$; fig. $3 C$ ). Drainage area $A$ and ecological diameter $l$ (fig. A1), two regional network descriptors ("Material and Methods"), did not significantly affect $\alpha$-diversity (fig. 3D, $3 E)$.

\section{Evenness Patterns}

In Riv landscapes, LC evenness depended significantly on patch size $\left(P_{\text {Riv }}=.004\right.$; fig. $\left.3 G\right)$. There was also a significant interaction of patch size and distance to the outlet in determining local evenness $\left(P_{\mathrm{Riv}}=.02\right.$; table A5). Instead, LC evenness in Ran and Hom landscapes did not show any significant dependence on any of the network descriptors (fig. 3F-3L; table A5). Importantly, the de- pendence of LC evenness on patch size in Riv landscapes at the local scale contributed to shape significantly higher evenness values at the regional scale, compared to values in Ran landscapes (paired $t$-test, $t_{4}=2.95, P=.041$; fig. $4 A$ ) or in Hom landscapes (paired $t$-test, $t_{4}=3.36, P=$ .028; fig. 4A). A higher variability in terms of coefficient of variation was detected in Riv landscapes across the five replicates (inset, fig. 4B). In Riv landscapes, species that presented low numbers of individuals in pure cultures, with higher body sizes and lower intrinsic growth rates, persisted at higher densities (figs. 4B, A3, B2).

\section{$\beta$-Diversity Patterns}

In Riv landscapes, $\beta_{\mathrm{MG}}$ increased with increasing pairwise topological distance between community pairs. No such pattern was found in the two other landscapes, and $\beta$ diversity showed a flat behavior (fig. $5 A$ ). Differences in community composition did not significantly depended on topological distance: Mantel tests revealed that there was no spatial dependence of $\beta$-diversity in any of the three configurations (figs. $5 A, \mathrm{Cl}$; table $\mathrm{C} 1$; fig. $\mathrm{C} 1$ and table $\mathrm{C} 1$ available online). Changes in patch size, instead, significantly affected $\beta$-diversity in Riv and Ran landscapes (figs. $5 B, \mathrm{C} 1$; table $\mathrm{C} 1$ ). Changes in ecological diameter, capturing the difference in network positioning (fig. A1) had a significant effect on $\beta$-diversity only in Riv and Hom landscapes (figs. $5 C, \mathrm{C} 1$; table $\mathrm{C} 1, \bar{m}_{\text {Riv }}=0.74, \bar{m}_{\text {Ran }}=$ $\left.0.16, \bar{m}_{\text {Hот }}=0.59\right)$.

\section{Discussion}

Our aquatic microcosm experiment disentangled the interaction between dendritic connectivity and hierarchical patch size on biodiversity of communities and showed that biodiversity patterns in river-like metacommunities significantly depend on the spatial covariance between dendritic connectivity, patch size, and position along the network.

\section{$\alpha$-Diversity Patterns}

Highly connected nodes, irrespective of the local environmental factors such as patch size, disturbances, and interspecific competition among species, sustained higher levels of local species richness compared to more peripheral communities (Finn et al. 2011; Carrara et al. 2012; Perkin and Gido 2012). Such a correlation is predicted by network theory (Newman 2010), where individuals on a random walk will be found in community $i$ proportionally to the degree of connectivity of $i$ on a connected network. In all three landscape configurations (fig. 1), $\alpha$-diversity increased with increasing connectivity. Similar results are 


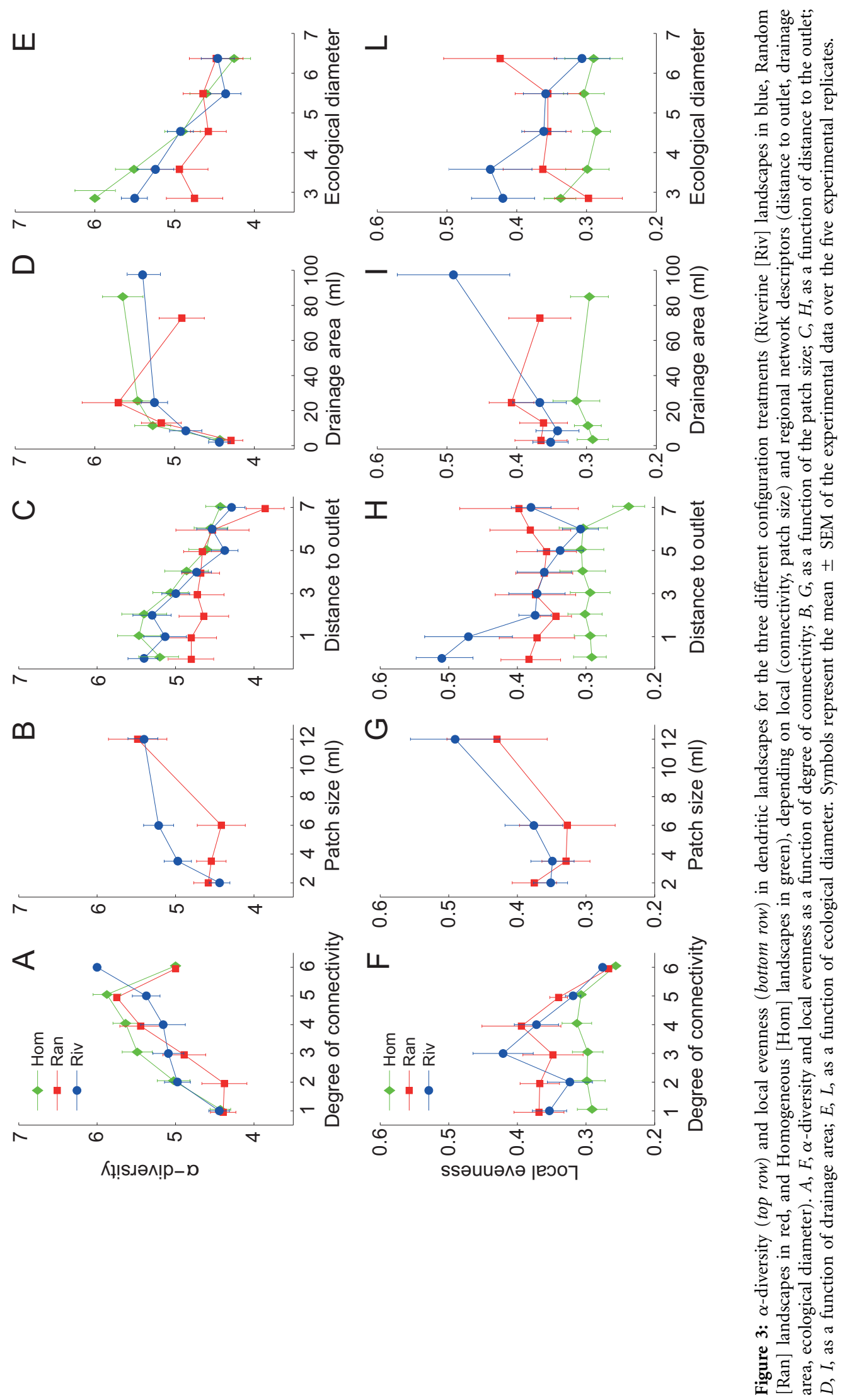

This content downloaded from 152.088.140.160 on August 11, 2017 02:27:10 AM

All use subject to University of Chicago Press Terms and Conditions (http://www.journals.uchicago.edu/t-and-c). 

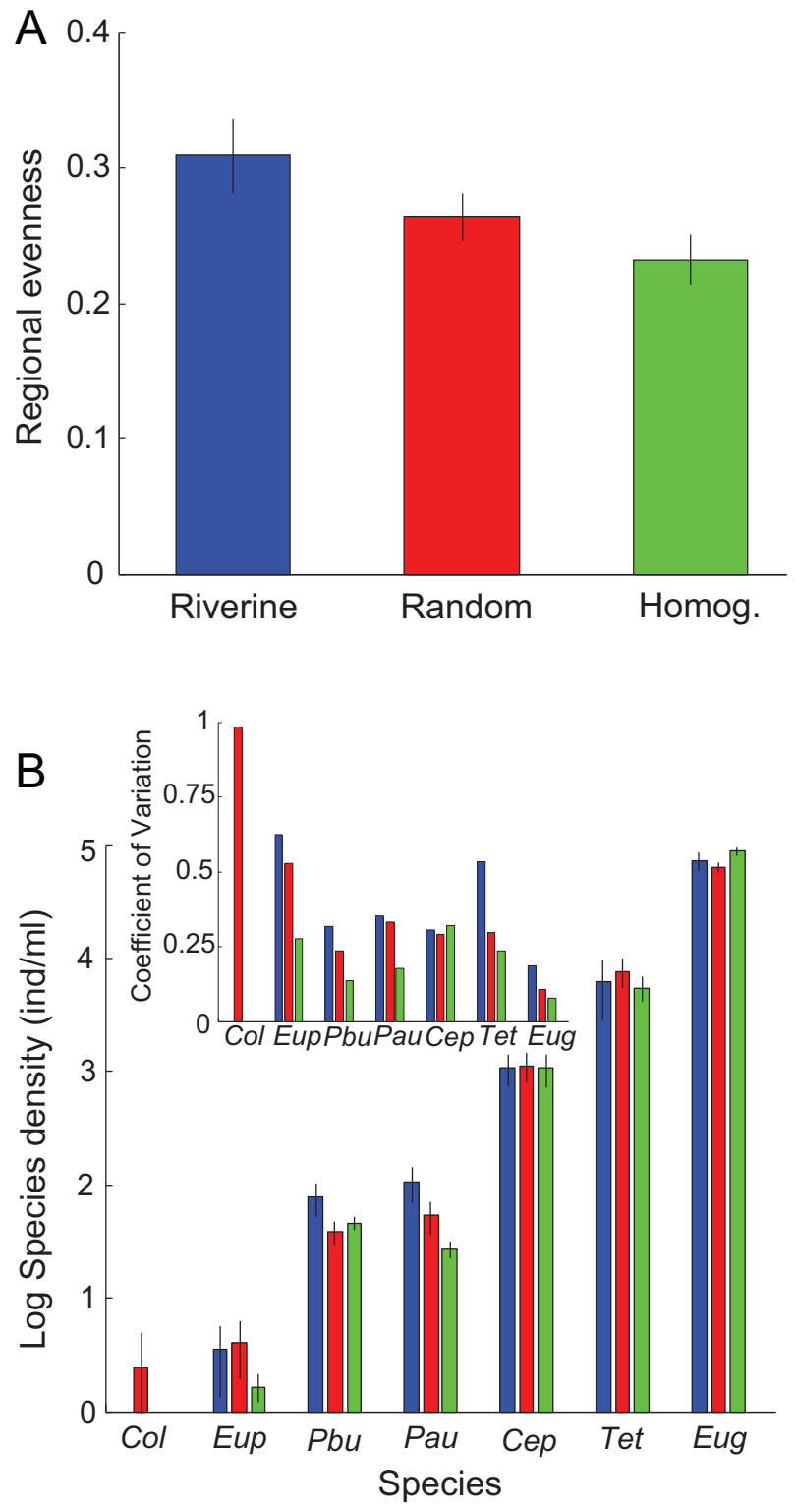

Figure 4: Community composition at metacommunity scale in Riverine (Riv; blue), Random (Ren; red), and Homogeneous (Hom; green) configurations. $A$, Regional evenness was significantly higher in Riv landscapes compared to Ran and Hom landscapes (average \pm SEM over the five experimental replicates). $B$, Species' density of the seven species detected at the end of the experiment for the three landscape configurations, sorted in increasing order of abundances (log scale). Values represent the mean \pm SEM across the five replicated landscapes. Inset, coefficient of variation for the same species over the five replicates $(\mathrm{Col}=$ Colpidium sp., Eup $=$ Euplotes aediculatus, $\mathrm{Pau}=$ Paramecium aurelia, $\mathrm{Pbu}=$ Paramecium bursaria, Cep = Cephalodella sp., Tet = Tetrahymena sp., and Eug = Euglena gracilis). obtained from a neutral model employed in spatially explicit networks (Economo and Keitt 2010). Neutral models focus on the spatial structure and dispersal limitation constrained by the specific landscape connectivity (Economo and Keitt 2008; Muneepeerakul et al. 2008; Economo and Keitt 2010), suggesting that dispersal in our experiment was a driving factor of $\alpha$-diversity.

In Riv landscapes, a consistent increase in $\alpha$-diversity was observed for larger patch sizes. Overall, in hierarchical Riv environments patch size is not significant because of the spatial covariance between patch size with the other network descriptors (table A1), which better describe the variation in local species richness. Only in Ran landscapes patch size, controlling formation, and composition of microhabitats (app. B), significantly affected $\alpha$-diversities. Our results show that species richness in dendritic landscapes with spatially uncorrelated patch size was determined largely by local drivers (i.e., degree of connectivity and patch size). The significant interaction between degree of connectivity and patch size in Ran landscapes (table A4) is suggesting that in intermediate patches (patch size 3.5 and $6 \mathrm{~mL}$ ), or in linear branches (connectivity $d=$ 2), the detrimental effects of disturbances (i.e., mortality due to emigration) on local species richness are enhanced (fig. $3 A, 3 B$ ). In Ran landscapes, the largest patches sustained higher abundances and thereby maintained a high $\alpha$-diversity irrespective of position (fig. $3 B$ ), as found in aquatic bacterial communities in mountain lakes (Reche et al. 2005). Previous protist studies identified species' specific responses to disturbances (Haddad et al. 2008; Carrara et al. 2012), and found that a high intrinsic growth rate is the most important factor in promoting a species' ability to survive a disturbance (Haddad et al. 2008). Such model systems cover substantial biological complexity in terms of species interactions and trophic levels that cannot be entirely captured by any model (app. B). Species with low reproductive number are prone to suffer more from environmental disturbances and require larger and well-connected habitats to persist (Staddon et al. 2010; Perkin and Gido 2012). In our experiment, Euplotes aediculatus, Paramecium aurelia, and Paramecium bursaria have lower intrinsic growth rates and lower carrying capacities in pure cultures (Altermatt et al. 2011a; Carrara et al. 2012). Because absolute numbers of individuals are lower for these species, demographic stochasticity plays an important role in affecting populations dynamics. In Ran landscapes, the negative effect of disturbances related to emigration on the survival of species with low reproductive rates was aggravated by the alteration of the natural hierarchical patch size configuration (see also app. A).

Regional network descriptors, that is, drainage area and ecological diameter (fig. A1), provide a synthesis of the above dynamics (fig. $3 D, 3 E$ ). In Riv and Hom landscapes, 

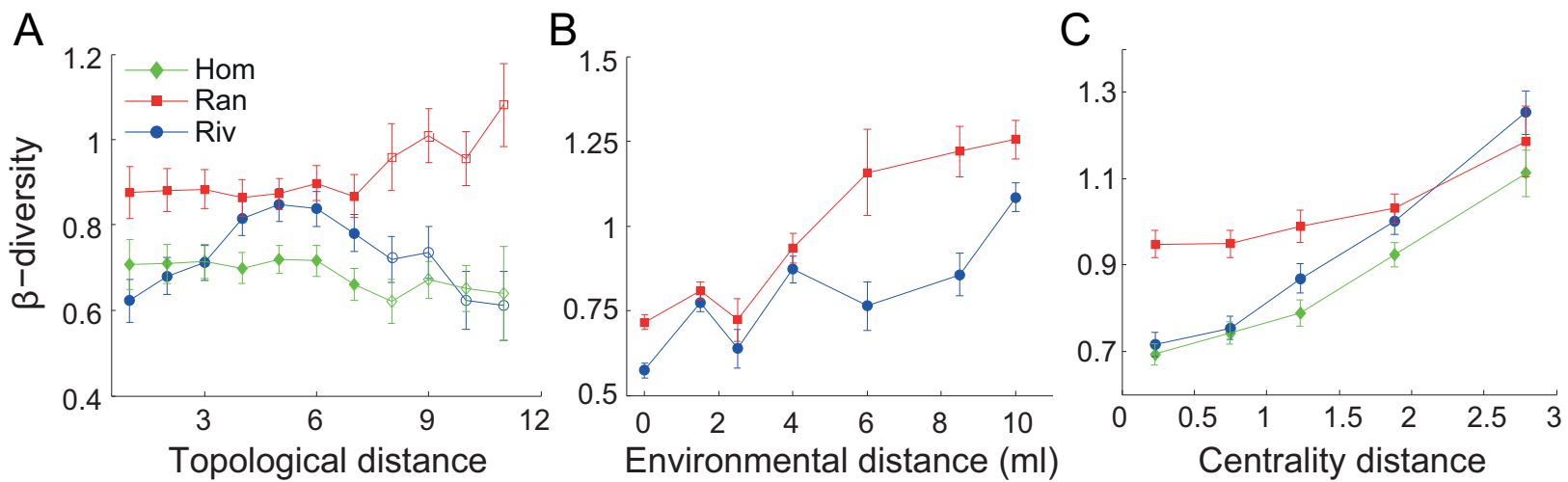

Figure 5: $\beta$-diversity as a function of the topological $(A)$, environmental $(B)$, and centrality distance $(C)$ between local community pairs, in Riverine (Riv), Random (Ran), and Homogeneous (Hom) configurations of patch size. Symbols represent the mean \pm SEM of the $\beta_{\mathrm{MG}}$-diversity data over the five experimental replicates. Open symbols in panel $(A)$ indicate the points where the statistical significance is lower (topological distance $\geq 8$; fig. $\mathrm{C} 1$, available online).

$\alpha$-diversity increased with increasing contributing drainage area. In Ran landscapes, we instead observed a local peak at intermediate values of total contributing area with a decline in $\alpha$-diversity for the largest values (fig. 3D). A likely explanation for this pattern is that in Ran metacommunities high-capacity patches are displaced also in peripheral zones of the network (fig. $1 B$ ), thereby acting as local sources of immigrants for the neighboring communities in the sub-basin. Through mass effects, the displaced high-capacity patches maintained species populations in the less favorable small or peripheral habitats (fig. $3 B$ ). In general, such mechanism of population survival is important in determining metapopulation dynamics (Holyoak et al. 2005). For example, in a plant pollinator system exposed to habitat fragmentation, it was found that highquality patches with large size were essential for bees persistence (Franzen and Nilsson 2010). It is a characteristic of natural riverine landscapes that high-capacity communities are placed at network position that have a high closeness centrality (Fernandes et al. 2004; fig. A1, table A1). These populations are thus strongly affecting the overall MC dynamics (Muneepeerakul et al. 2007; fig. 3E). Here, we experimentally demonstrated that breaking the natural link between patch size and connectivity at local scales destroys the regional pattern in species richness observed in Riv and Hom landscapes. In Ran configuration, local species richness did not increase while approaching the outlet community (fig. $3 C$ ), despite the converging character of the network structure and downstream-biased dispersal (Muneepeerakul et al. 2008). This suggests that in rivers the positioning of high-capacity patches has a higher significance for biodiversity (Lowe et al. 2006; Grant et al. 2007) than in other types of landscapes, such as ponds, islands, or lakes (De Bie et al. 2012). Even though habitat capacity and interannual streamflow variability, which are altered in rivers undergoing hydropower development (Poff et al. 2007; Grant et al. 2012; Ziv et al. 2012), may not exactly correspond to our Ran landscapes, we here provide proof of principles for how altering the hierarchical patch size configuration in dendritic systems may impact on important biodiversity patterns of aquatic microbial communities.

\section{Community Composition}

Locally (i.e., at the patch scale), variation in patch size configuration in dendritic networks altered community composition. Theory suggests that species that are better competitors in a particular environmental condition eventually spread along the system, impeding other species' growth and thus exposing them to higher extinction levels (Hillebrand et al. 2008; Cardinale 2011). Accordingly, in our experiment we observed a predominance of Euglena gracilis (best competitor of our species pool) in Hom landscapes, whereas populations of larger species (E. aediculatus, $P$. aurelia, and $P$. bursaria) were reduced compared to heterogeneous configurations (fig. $4 B$ ). We found that habitat heterogeneity in Riv and Ran landscapes promoted both local species evenness (fig. $2 B$ ) and persistence of $\beta$-diversity (fig. $2 C$ ), compared to Hom landscapes. This gives a causal, experimental proof of principles on how homogenization of habitat size along river networks can affect diversity (Lowe et al. 2006).

At regional spatial scales, the consequences of the spatial configuration of patch sizes on community composition in terms of degree of dominance and species turnover were subtle. Only in Riv landscapes, LC evenness increased consistently with increasing patch size and decreasing distance 
to the outlet (fig. $3 G, 3 H$ ). This highlights the structuring power of hierarchy (fig. 3I, 3L). Spatial environmental autocorrelation resulted in higher levels of regional evenness in Riv ecosystems (fig. 4A), by increasing the population size of some of the rarer species and at the same time decreasing the total biomass of the more abundant species (figs. $4 B, \mathrm{~B} 2$ ). Possibly, rare species with lower growth rates were able to track their specific niche requirements more efficiently in Riv landscapes (Cardinale 2011). Thereby, they grew to higher population densities compared to the Ran landscapes where the spatial autocorrelation was disentangled (see app. A, figs. A2-A5, where a stochastic metacommunity model is presented, generalizing this finding). We demonstrated how alterations of river-like landscapes may have strong effects on MC dynamics and impact important regional diversity and evenness properties (Hillebrand et al. 2008). We also found that the modified Gower's $\beta_{\mathrm{MG}}$ was mostly driven by its abundance component $\beta_{\mathrm{A}}$ (inset, fig. $2 C$ ), indicating that empirical and theoretical studies in community ecology and conservation biology have to consider species abundances.

The $\beta$-diversity increased in Riv landscapes with increasing topological distance along the network (fig. 5A). Such a pattern is commonly observed in comparative studies on riverine diversity (Muneepeerakul et al. 2008; Brown and Swan 2010). No spatial correlation of LC similarity was found in Ran and Hom landscapes (fig. $5 A$ ), suggesting that a combination of patch size and network position is needed to reproduce this pattern. The flat behavior of $\beta$-diversity against topological distance reflected the prominent role of local environmental conditions in structuring communities through species sorting and competition. Dispersal limitation alone cannot reproduce such an effect on community differentiation (Brown and Swan 2010; Astorga et al. 2012). We argue that priority effects, reflecting colonization history, did not play an important role in community assembly as species were present everywhere at the start of the experiment. Randomizing patch size, that is, altering the hierarchical riverine structure, is an analog of fragmenting the landscapes (see effects on $\alpha$-diversity: fig. $3 A, 3 B, 3 D)$. At the same time it is opening up more diversified spatiotemporal niches (Chesson 2000) and producing more distinct species compositions. Interestingly, $\beta$-diversity depended on centrality in the Hom landscapes with fixed habitat capacity. This strongly indicates that dendritic connectivity per se shapes both $\alpha$-and $\beta$-diversity (Carrara et al. 2012). Communitycomposition turnover along centrality gradient was maximized for Riv landscapes, and hierarchical patch size distribution enhanced the turnover provided by dendritic connectivity itself (fig. $5 C$; table $\mathrm{C} 1$ ).

Our previous work, which was done with a similar model system, focused only on effect of connectivity (Carrara et al. 2012). This study implemented a more realistic realization of natural rivers with varied patch size and biased dispersal kernel (Fagan 2002; Grant et al. 2007; Muneeperakul 2007). We disentangled for the first time the effects of the intrinsic link of network position and habitat patch size on diversity and community evenness and also looked at effects on common versus rare species by altering riverine structure. We indeed provided evidence of the interaction between species traits and population responses to spatiotemporal gradients of local environmental conditions in spatially structured habitats. For example, fast population growth, allowing rapid population responses to a more unpredictable environment, might favor species with higher intrinsic growth rates and vice versa. Our protist species are naturally co-occuring in freshwater habitats and cover a wide range of intrinsic growth rates, body sizes, and other important biological traits, such as dispersal ability (Altermatt et al. 2011a; Carrara et al. 2012). We stress that our experiments, as in similar model systems (Cadotte et al. 2006; Haddad et al. 2008; Livingston et al. 2012), are conceptualized versions of natural ecosystems and do not allow direct extrapolation of our results to natural rivers. However, they enhance our understanding of complex systems in nature, where multiple processes are interacting on different scales (Holyoak and Lawler 2005). Dispersal rate, dispersal mode, and the strength of directionality are important factors in determining community patterns in theoretical models (Mouquet and Loreau 2003; Morrissey and de Kerckhove 2009) and natural communities (Clarke et al. 2008). In analogy to our experiments, recent comparative studies (Brown and Swan 2010; Astorga et al. 2012; De Bie et al. 2012; Heino 2013) showed that different dispersal abilities, controlled by body size and dispersal mode, determine a change in the response of community similarity to environmental variation and geographic distance. This suggests that the relative importance of the two structuring forces may depend on the group of organisms and the spatial scale. In riverine ecosystems, the river network itself provides at the same time the primary habitat for the species and suitable ecological corridors for individuals to disperse (Fagan 2002; Grant et al. 2007; Rodriguez-Iturbe et al. 2009), resulting in a close match between the physical and the ecological scales (Grant et al. 2007). This correspondence is recognized as important for the ecosystems' resilience at different levels of ecological complexity (Gonzalez et al. 2011). For a variety of species living in natural riverine systems, out-of-network movements are likely to occur, leading to intercatchment dispersal (Clarke et al. 2008; Brown et al. 2010). In macroinvertebrates, active dispersers with a terrestrial stage should track environmental heterogeneity better than passive dispersers with 
only an aquatic stage (Heino 2013). Moreover, the strength of directionality in river systems might be much stronger for passive dispersers, as bacteria and protists, compared to macroinvertebrates, amphibians, or fishes (Astorga et al. 2012; De Bie et al. 2012). For example, a neutral metacommunity model showed that a symmetric dispersal kernel suitably described fishes' biodiversity patterns in the Mississippi-Missouri river system (Muneepeerakul et al. 2008). Thus, when comparing or extrapolating our results to natural systems, one needs to carefully assume that taxon specific aspect of dispersal are fulfilled and patterns and processes may not scale directly across all species and landscapes sizes. In our experiment, adopting a diffusive downstream-biased dispersal between isolated habitat patches and at discrete-time intervals ("Material and Methods"), different dispersal strategies could not naturally arise. Competition-colonization trade-offs were documented in protist studies that were adopting similar species (Cadotte et al. 2006; but see Haddad et al. 2008). Such mechanism of species coexistence, together with the storage effect (Chesson 2000), may interact with the spatial structure to shape diversity and ecosystem productivity, as tested in bacterial metacommunities (Livingston et al. 2012). As the system was continuously perturbed away from stationarity by dispersal and emigration mortality, we were observing a transient state. The duration of transient dynamics in our networks may depend nontrivially on the different patch sizes, which are sustaining different population sizes. System relaxation time to equilibrium could be investigated by implementing a metacommunity model with salient features of our experiment, but it goes beyond the scope of the present study.

\section{Conclusions}

Understanding the effects of fragmentation and patch size distribution on communities is crucial, especially in highly diverse riverine systems. Hierarchical riverine habitats, characterized by a natural spatiotemporal heterogeneity, sustain higher levels of diversity (Muneepeerakul et al. 2007; Carrara et al. 2012). Because dispersal is constrained by the network pathway, the river network may become a trap for species when the dendritic system is exposed to habitat fragmentation and path size alterations. Protecting highly connected communities could help to avoid extinctions of species with low reproductive rates. These species are prone to suffer more from environmental disturbances and require larger and well-connected habitats to persist. By preserving the natural hierarchy of spatiotemporal heterogeneity along river networks, fast growing species and weak competitors alike are better able to persist. Our results not only causally demonstrate general ecological principles, but also give insights for developing the- oretical metacommunity models in dendritic environments and for future empirical studies focusing on riverine ecosystems.

\section{Acknowledgments}

We thank J. Chase, M. Vellend, and four anonymous reviewers for useful comments and suggestions on a previous version of the manuscript. We are grateful to E. Bertuzzo, M. Gatto, and L. Mari for interesting discussions. We acknowledge F. de Alencastro (Central Environmental Analytical Laboratory, Central Engineering Institute, École Polytechnique Fédérale Lausanne) for his generous support and for the access to his laboratory material. Funding from European Research Council Advanced Grant (River Networks as Ecological Corridors) 22761 (A.R., F.C.) and Swiss National Science Foundation grants 200021/124930/ 1 (A.R., F.C.) and 31003A-135622 (A.G., F.A.).

\section{Literature Cited}

Altermatt, F., A. Bieger, F. Carrara, A. Rinaldo, and M. Holyoak. 2011a. Effects of connectivity and recurrent local disturbances on community structure and population density in experimental metacommunities. PLoS ONE 6:e19525. doi:10.1371/journal .pone.0019525.

Altermatt, F., V. I. Pajunen, and D. Ebert. 2008. Climate change affects colonization dynamics in a metacommunity of three daphnia species. Global Change Biology 14:1209-1220.

Altermatt, F., S. Schreiber, and M. Holyoak. 2011b. Interactive effects of disturbance and dispersal directionality on species richness and composition in metacommunities. Ecology 92:859-870.

Anderson, M., K. Ellingsen, and B. McArdle. 2006. Multivariate dispersion as a measure of beta diversity. Ecology Letters 9:683-693.

Anderson, M. J., T. O. Crist, J. M. Chase, M. Vellend, B. D. Inouye, A. L. Freestone, N. J. Sanders, et al. 2011. Navigating the multiple meanings of beta diversity: a roadmap for the practicing ecologist. Ecology Letters 14:19-28.

Astorga, A., J. Oksanen, M. Luoto, J. Soininen, R. Virtanen, and T. Muotka. 2012. Distance decay of similarity in freshwater communities: do macro- and microorganisms follow the same rules? Global Ecology and Biogeography 21:365-375.

Banavar, J. R., J. Damuth, A. Maritan, and A. Rinaldo. 2007. Scaling in ecosystems and the linkage of macroecological laws. Physical Review Letters 98:068104.

Benda, L., N. L. Poff, D. Miller, T. Dunne, G. Reeves, G. Pess, and M. Pollock. 2004. The network dynamics hypothesis: how channel networks structure riverine habitats. BioScience 54:413-427.

Brown, B. L., and C. M. Swan. 2010. Dendritic network structure constrains metacommunity properties in riverine ecosystems. Journal of Animal Ecology 79:571-580.

Cadotte, M. W., D. V. Mai, S. Jantz, M. D. Collins, M. Keele, and J. A. Drake. 2006. On testing the competition-colonization trade-off in a multispecies assemblage. American Naturalist 168:704-709. 
Cardinale, B. J. 2011. Biodiversity improves water quality through niche partitioning. Nature 472:86-91.

Carrara, F., F. Altermatt, I. Rodriguez-Iturbe, and A. Rinaldo. 2012. Dendritic connectivity controls biodiversity patterns in experimental metacommunities. Proceedings of the National Academy of Sciences of the USA 109:5761-5766.

Carrara, F., A. Rinaldo, A. Giometto, and F. Altermatt. 2013. Data from: Complex interaction of dendritic connectivity and hierarchical patch size on biodiversity in river-like landscapes. American Naturalist, Dryad Digital Repository, http://dx.doi.org/10.5061 /dryad.15np2.

Chesson, P. 2000. General theory of competitive coexistence in spatially-varying environments. Theoretical Population Biology 58: 211-237.

Chisholm, C., Z. Lindo, and A. Gonzalez. 2011. Metacommunity diversity depends on network connectivity arrangement in heterogeneous landscapes. Ecography 34:415-424.

Clarke, A., R. Mac Nally, N. Bond, and P. S. Lake. 2008. Macroinvertebrate diversity in headwater streams: a review. Freshwater Biology 53:1707-1721.

De Bie, T., L. De Meester, L. Brendonck, K. Martens, B. Goddeeris, D. Ercken, H. Hampel, et al. 2012. Body size and dispersal mode as key traits determining metacommunity structure of aquatic organisms. Ecology Letters 15:740-747.

Economo, E. P., and T. H. Keitt. 2008. Species diversity in neutral metacommunities: a network approach. Ecology Letters 11:52-62.

- 2010. Network isolation and local diversity in neutral metacommunities. Oikos 119:1355-1363.

Fagan, W. F. 2002. Connectivity, fragmentation, and extinction risk in dendritic metapopulations. Ecology 83:3243-3249.

Fahrig, L. 2003. Effects of habitat fragmentation on biodiversity. Annual Review of Ecology Evolution and Systematics 34:487-515.

Fernandes, C. C., J. Podos, and J. G. Lundberg. 2004. Amazonian ecology: tributaries enhance the diversity of electric fishes. Science 305:1960-1962.

Finn, D. S., N. Bonada, C. Murria, and J. M. Hughes. 2011. Small but mighty: headwaters are vital to stream network biodiversity at two levels of organization. Journal of the Northern American Benthological Society 30:963-980.

Franzen, M., and S. G. Nilsson. 2010. Both population size and patch quality affect local extinctions and colonizations. Proceedings of the Royal Society B: Biological Sciences 277:79-85.

Fukami, T., and P. J. Morin. 2003. Productivity-biodiversity relationships depend on the history of community assembly. Nature 424:423-426.

Gassner, A. 2006. Gewässerschutzbestimmungen in der Landwirtschaft: ein internationaler vergleich (Bundesamt für Umwelt, Bern. 76 S.). Umwelt-Wissen 0618.

Giometto, A., F. Altermatt, F. Carrara, A. Maritan, and A. Rinaldo. 2013) Scaling body-size fluctuations. Proceedings of the National Academy of Sciences of the USA 110:4646-4650.

Gonzalez, A., J. H. Lawton, F. S. Gilbert, T. M. Blackburn, and I. Evans-Freke. 1998. Metapopulation dynamics, abundance, and distribution in a microecosystem. Science 281:2045-2047.

Gonzalez, A., B. Rayfield, and Z. Lindo. 2011. The disentangled bank: how loss of habitat fragments and disassembles ecological networks. American Journal of Botany 98:503-516.

Grant, E. H. C., W. H. Lowe, and W. F. Fagan. 2007. Living in the branches: population dynamics and ecological processes in dendritic networks. Ecology Letters 10:165-175.
Grant, E. H. C., H. J. Lynch, R. Muneepeerakul, M. Arunachalam, I. Rodriguez-Iturbe, and W. F. Fagan. 2012. Interbasin water transfer, riverine connectivity, and spatial controls on fish biodiversity. PLoS ONE 7:e34170.

Haddad, N. M., M. Holyoak, T. M. Mata, K. F. Davies, B. A. Melbourne, and K. Preston. 2008. Species traits predict the effects of disturbance and productivity on diversity. Ecology Letters 11:348356.

Heino, J. 2013. Environmental heterogeneity, dispersal mode, and co-occurrence in steream macroinvertebrates. Ecology and Evolution 3:344-355.

Hillebrand, H., D. M. Bennett, and M. W. Cadotte. 2008. Consequences of dominance: a review of evenness effects on local and regional ecosystem processes. Ecology 89:1510-1520.

Holyoak, M., and S. P. Lawler. 2005. The contribution of laboratory experiments on protists to understanding population and metapopulation dynamics. Advances in Ecological Research 37:245271.

Holyoak, M., M. A. Leibold, and R. D. Holt. 2005. Metacommunities: spatial dynamics and ecological communities. University of Chicago Press, Chicago.

Hubbell, S. P. 2001. The unified theory of biodiversity and biogeography. Princeton University Press, Princeton, NJ.

Lake, P. S., N. Bond, and P. Reich. Linking ecological theory with stream restoration. 2007. Freshwater Biology 52:597-615.

Leopold, L. B., M. G. Wolman, J. P. and Miller. 1964. Fluvial processes in geomorphology. Freeman, San Francisco.

Livingston, G., M. Matias, V. Calcagno, C. Barbera, M. Combe, M. A. Leibold, and N. Mouquet. 2012. Competition-colonization dynamics in experimental bacterial metacommunities. Nature Communications 3:1234. doi:10.1038/ncomms2239.

Lowe, W. H., G. E. Likens, and M. E. Power. 2006. Linking scales in stream ecology. BioScience 56:591-597.

MacArthur, R. H., and E. O. Wilson. 1963. Equilibrium-theory of insular zoogeography. Evolution 17:373-387.

Matthiessen, B., and H. Hillebrand. 2006. Dispersal frequency affects local biomass production by controlling local diversity. Ecology Letters 9:652-662.

Morrissey, M. B., and D. T. de Kerckhove. 2009. The maintenance of genetic variation due to asymmetric gene flow in dendritic metapopulations. American Naturalist 174:875-889.

Mouquet, N., and M. Loreau. 2003. Community patterns in sourcesink metacommunities. American Naturalist 162:544-557.

Muneepeerakul, R., E. Bertuzzo, H. J. Lynch, W. F. Fagan, A. Rinaldo, and I. Rodriguez-Iturbe. 2008. Neutral metacommunity models predict fish diversity patterns in Mississippi-Missouri basin. Nature 453:220-222.

Muneepeerakul, R., J. S. Weitz, S. A. Levin, A. Rinaldo, and I. Rodriguez-Iturbe. 2007. A neutral metapopulation model of biodiversity in river networks. Journal of Theoretical Biology 245:351363.

Newman, M. E. J. 2010. Networks. Oxford University Press, Oxford. Perkin, P. J., and K. B. Gido. 2012. Fragmentation alters stream fish community structure in dendritic ecological networks. Ecological Applications 22:2176-2187.

Poff, N. L., J. D. Olden, D. M. Merritt, and M. Pepin. 2007. Homogenization of regional river dynamics by dams and global biodiversity implications. Proceedings of the National Academy of Sciences of the USA 104:5732-5737.

Reche I., E. Pulido-Villena, R. Morales-Baquero, and E. O. Casa- 
mayor. 2005. Does ecosystem size determine aquatic bacterial richness? Ecology 86:1715-1722.

Rinaldo, A., J. R. Banavar, and A. Maritan. 2006. Trees, networks and hydrology. Water Resources Research 42:W06D07.

Rinaldo, A., I. Rodriguez-Iturbe, R. Rigon, R. L. Bras, E. Ijjasz-Vasquez, and A. Marani. 1992. Minimum energy and fractal structures of drainage networks. Water Resources Research 28:2183-2191.

Rodriguez-Iturbe, I., R. Muneepeerakul, E. Bertuzzo, S. A. Levin, and A. Rinaldo. 2009. River networks as ecological corridors: a complex systems perspective for integrating hydrologic, geomorphologic, and ecologic dynamics. Water Resources Research 45:W01413.

Rodriguez-Iturbe, I., and A. Rinaldo. 1997. Fractal river basins: chance and self-organization. Cambridge University Press, New York.

Rodriguez-Iturbe, I., A. Rinaldo, R. Rigon, R. L. Bras, and E. IjjaszVasquez. 1992. Energy dissipation, runoff production and the three dimensional structure of channel networks. Water Resources Research 28:1095-1103.

Sheldon, A. L. 1968. Species diversity and longitudinal succession in stream fishes. Ecology 49:193-198.

Smith, B., and J. Wilson. 1996. A consumer's guide to evenness indices. Oikos 76:70-82.

Staddon, P., Z. Lindo, P. D. Crittenden, F. Gilbert, and A. Gonzalez.
2010. Connectivity, non-random extinction and ecosystem function in experimental metacommunities. Ecology Letters 13:543552.

Urban, M. C., D. K. Skelly, D. Burchsted, W. Price, and S. Lowry. 2006. Stream communities across a rural-urban landscape gradient. Diversity and Distributions 12:337-350.

Vannote, R. L., G. W. Minshall, K. W. Cummins, J. R. Sedell, and C. E. Cushing. 1980. River continuum concept. Canadian Journal of Fisheries and Aquatic Sciences 37:130-137.

Vörösmarty, C. J., P. B. McIntyre, M. O. Gessner, D. Dudgeon, A. Prusevich, P. Green, S. Glidden, et al. 2010. Global threats to human water security and river biodiversity. Nature 467:555-561.

Warren, P. H. 1996. Dispersal and destruction in a multiple habitat system: an experimental approach using protist communities. Oikos 77:317-325.

Ziv, G., E. Baran, S. Nam, I. Rodriguez-Iturbe, and S. A. Levin. 2012. Trading-off fish biodiversity, food security, and hydropower in the Mekong River Basin. Proceedings of the National Academy of Sciences of the USA 109:5609-5614.

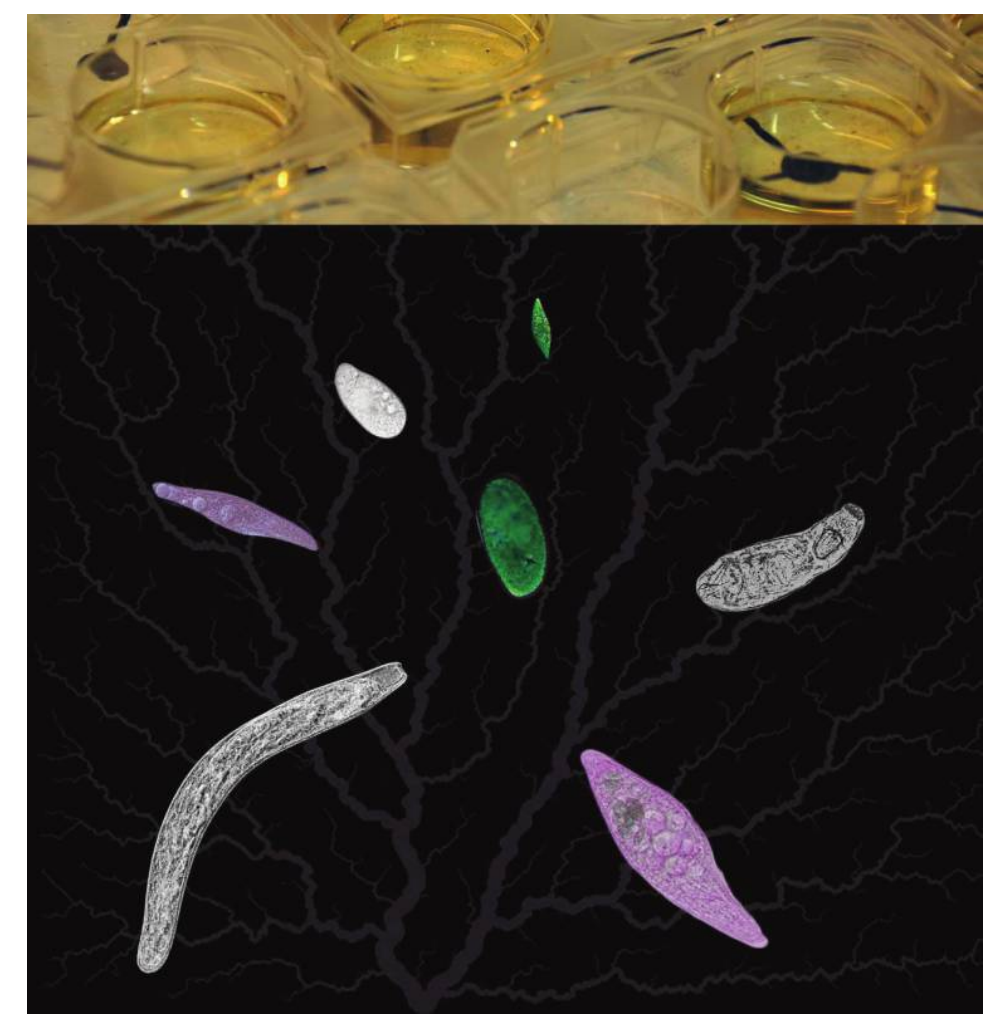

Connectivity and habitat size rule community composition in riverine landscapes. Some of the protist species used in the experiment (from the top, counterclockwise): Euglena gracilis (green), Colpidium sp. (white), Blepharisma sp. (pink), Spirostomum sp. (black and white), Blepharisma (again in pink), Cephalodella sp. (black and white), and in the center, Paramecium bursaria (green). Photo credit: Francesco Carrara and Regula Illi. 